\title{
Electronic and Magnetic Properties of Co-doped $\mathrm{Rb}_{2} \mathrm{Ni}_{3} \mathrm{~S}_{4}$
}

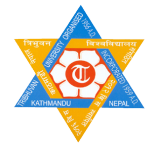

Gang Bahadur Acharya

Central Department of Physics

Tribhuvan University, Kirtipur, Nepal

\section{Supervisor}

Dr. Madhav Prasad Ghimire

Associate Professor

October 20, 2020 


\section{Outline}

- Introduction/Background

- Methodology and Computational tools

- Electronic and magnetic properties of $\mathrm{Rb}_{2} \mathrm{Ni}_{3} \mathrm{~S}_{4}$

- Half metallicity in Co-doped $\mathrm{Rb}_{2} \mathrm{Ni}_{3} \mathrm{~S}_{4}$

- Discussion and Conclusions 


\section{Introduction/Background}

Kagome Metals

- Kagome: Traditional Japanese woven bamboo pattern.

- Japanese Word : Kago - Basket, Me Eyes.

- Atoms of a conducting substances arranged in Kagome pattern - shows exotic electronic properties.

- Kagome lattice: Vertices and edges of tri-hexagonal tiling, each hexagon is surrounded by triangles.

- Importance : conduct electricity without losing energy at room temperature.

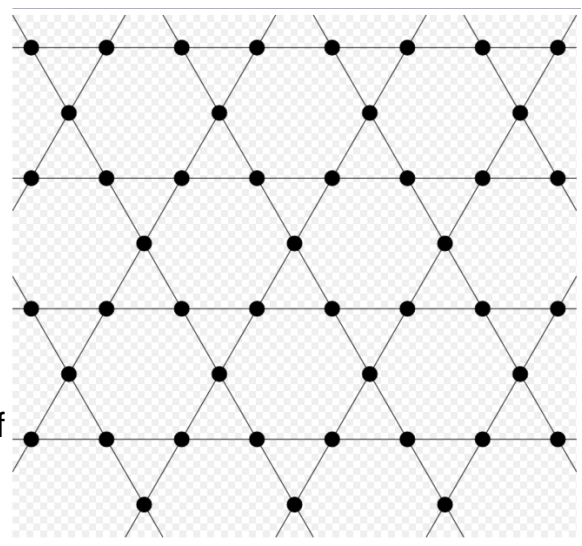

Figure: structure of kagome lattice.

a

${ }^{a}$ http://www.hfmphysics.com/2006/motif. 


\section{Introduction/Background}

Half Metals

- One spin channel metallic.

- Opposite spin channel insulating.

- Zero energy band gap superior electronic properties than non zero energy gap material.

- Importance: Practical applications in spintronics, electronics and sensors.

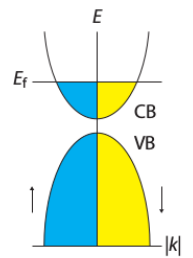

Metal

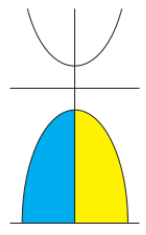

Semiconductor

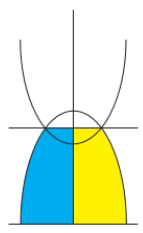

Semi-metal

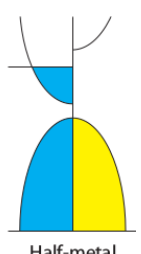

Half-metal

Figure: The electronic band structures of various classes of materials. ${ }^{1}$

${ }^{1}$ (Wang et al., NPG Asia Mater. 2, 31, (2010) 


\section{Crystal structure $\mathrm{Rb}_{2} \mathrm{Ni}_{3} \mathrm{~S}_{4}$}

- Face centered-orthorhombic structure with the space group Fmmm (69)

- Symmorphic space group

- lattice parameters: $a=$ $5.90615070 \AA, b=$ $10.06449278 \AA, c=$ $13.43457036 \AA$

Figure: Ni ions constitute a Kagome lattice
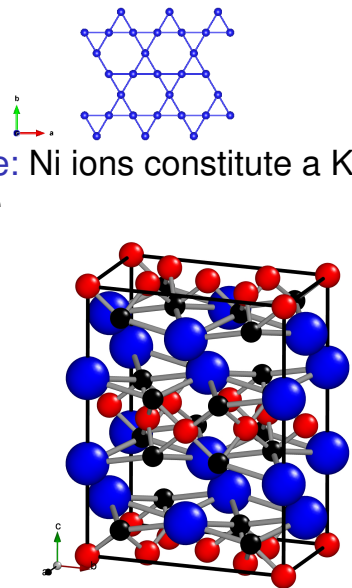

- Angles : $\alpha=\beta=\gamma=$ $90^{\circ}$

Figure: Crystal structure of $\mathrm{Rb}_{2} \mathrm{Ni}_{3} \mathrm{~S}_{4}$. (blue balls are $\mathrm{Rb}$ atoms, red balls are $\mathrm{Ni}$ atoms and black balls are $\mathrm{S}$ atoms) 


\section{Methodology and Computational tools}

- Study electronic and magnetic properties of $\mathrm{Rb}_{2} \mathrm{Ni}_{3} \mathrm{~S}_{4}$ and Co-doped $\mathrm{Rb}_{2} \mathrm{Ni}_{3} \mathrm{~S}_{4}$ Density functional theory (DFT)calculation.

- Generalized gradient approximation (GGA) used for exchange correlation interaction.

- Full Potential Local Orbital (FPLO)code used for calculations. 


\section{Results and Discussion}

Electronic and Magnetic Properties of $\mathrm{Rb}_{2} \mathrm{Ni}_{3} \mathrm{~S}_{4}$

- Nonmagnetic, ferromagnetic and antiferromagnetic configuration.

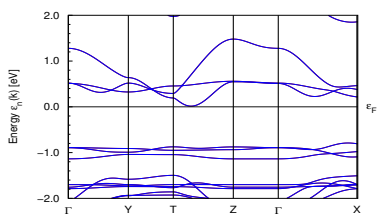

- Ground state is to be weak ferromagnetic.

Figure: Band structure of $\mathrm{Rb}_{2} \mathrm{Ni}_{3} \mathrm{~S}_{4}$ in scalar relativistic.

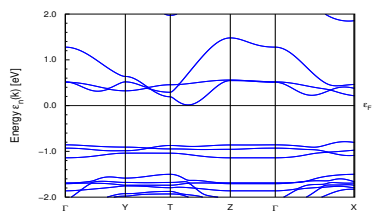

Figure: Band structure of $\mathrm{Rb}_{2} \mathrm{Ni}_{3} \mathrm{~S}_{4}$ in full relativistic. 


\section{Results and Discussion}

Electronic and Magnetic Properties of Co-doped $\mathrm{Rb}_{2} \mathrm{Ni}_{3} \mathrm{~S}_{4}$

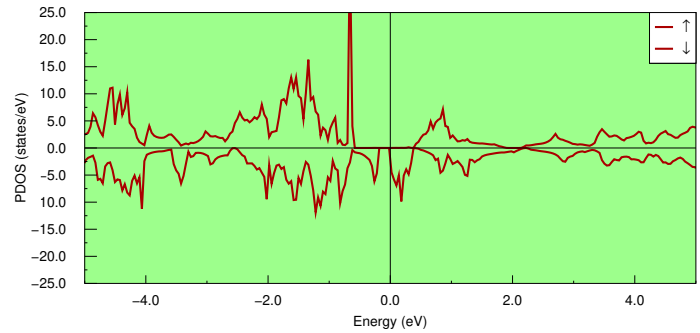

Figure: Density of states of Co-doped $\mathrm{Rb}_{2} \mathrm{Ni}_{3} \mathrm{~S}_{4}$ in scalar relativistic

- Co-doped in the place of first $\mathrm{Ni}$.

- Ground state is ferromagnetic.
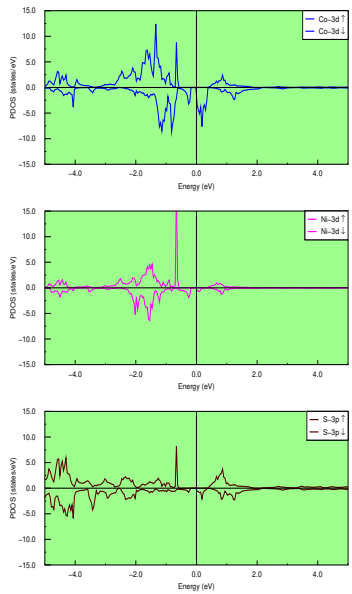

Figure: Partial Density of states of Co-doped $\mathrm{Rb}_{2} \mathrm{Ni}_{3} \mathrm{~S}_{4}$ in scalar relativistic. 


\section{Results and Discussion}

Electronic and Magnetic Properties of Co-doped $\mathrm{Rb}_{2} \mathrm{Ni}_{3} \mathrm{~S}_{4}$

- Half metallic ferromagnetism.

- magnetic moment $2.0 \mu_{B} /$ unit cell.

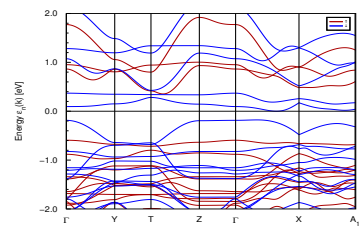

Figure: Band structure of Co-doped

- Ferromagnetism mainly derived from Co-3d spins.

- Strong hybridization between $\mathrm{Ni}-3 \mathrm{~d}$ and Co-3d orbitals.

$\mathrm{Rb}_{2} \mathrm{Ni}_{3} \mathrm{~S}_{4}$ in scalar relativistic

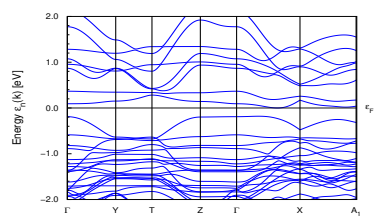

Figure: Band structure of Co-doped $\mathrm{Rb}_{2} \mathrm{Ni}_{3} \mathrm{~S}_{4}$ in full relativistic 


\section{Electronic and Magnetic Properties of Co-doped $\mathrm{Rb}_{2} \mathrm{Ni}_{3} \mathrm{~S}_{4}$}

- Kagome lattice materials can host flat band.

- With energy $0.36 \mathrm{eV}$ above the Fermi level.

- Electronic flat bands in momentum space arising from strong localization of electrons.

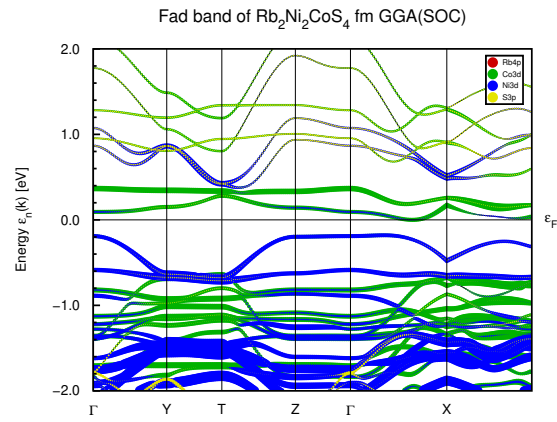

Figure: Fad band structure of Co-doped $\mathrm{Rb}_{2} \mathrm{Ni}_{3} \mathrm{CoS}_{4}$ in full relativistic 


\section{Conclusions}

- Parent materials $\mathrm{Rb}_{2} \mathrm{Ni}_{3} \mathrm{~S}_{4}$ is weak ferromagnetic in nature.

- Upon full replacement of $\mathrm{Ni}(1)$ by $\mathrm{Co}$ atom ferromagnetic half metallic state achieved.

- Strong hybridization between Ni 3d and Co 3d orbitals. 


\section{Acknowledgment}

- Assoc. Prof. Dr. Madhav Prasad Ghimire (Supervisor)

CDP, Nepal

- PD Dr. Manuel Richter (Co-supervisor)

IFW Dresden

- Prof. Dr. Binil Aryal (HOD) 


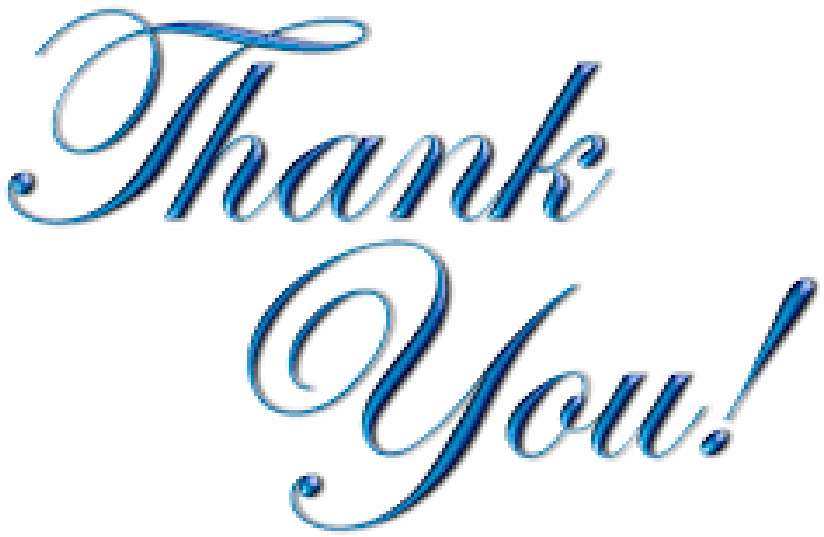

October 20, 2020

$13 / 13$ 